\title{
High serum concentrations of growth differentiation factor-15 in male Crohn's disease patients with low skeletal muscle index
}

Hiroyuki Yamamoto ( $\triangle$ yamamotsu.14@gmail.com )

Nagasaki University

Fuminao Takeshima

Nagasaki University

Masafumi Haraguchi

Nagasaki University

Kayoko Matsushima

Nagasaki University

Moto Kitayama

Nagasaki University

Yuko Akazawa

Nagasaki University

Kumi Ogihara

Nagasaki University

Maiko Tabuchi

Nagasaki University

Keiichi Hashiguchi

Nagasaki University Hospital

Naoyuki Yamaguchi

Nagasaki University Hospital

Kazuhiko Nakao

Nagasaki University

\section{Research Article}

Keywords: Crohn's disease, Sarcopenia, Growth Differentiation Factor-15 (GDF-15)

Posted Date: September 20th, 2021

DOI: https://doi.org/10.21203/rs.3.rs-904046/v1 
License: (c) (i) This work is licensed under a Creative Commons Attribution 4.0 International License. Read Full License 


\section{Abstract}

Sarcopenia is defined as low skeletal muscle index (SMI) in addition to low muscle strength (MS) or low physical function, and many biomarkers have been reported. In Crohn's disease (CD), low SMI is associated with predictors and complications of intestinal resection. Therefore, in many reports of CD, sarcopenia was defined only by SMI. However, there have been no reports of MS in Japan. Our study aimed to investigate the frequency of sarcopenia by assessing both SMI and MS in Japanese patients with $\mathrm{CD}$ and biomarkers predicting low SMI. We evaluated SMI using bioelectrical impedance analysis, handgrip strength, and blood tests, including CRP, ALB, IL-6, TNFa, GDF-8, and GDF-15 as biomarker candidates for $78 \mathrm{CD}$ patients in our hospital. Sarcopenia and low SMI were $8 \%$ and $42.3 \%$, respectively. Each candidate biomarker and SMI were negatively correlated with GDF-15 (Pearson's $r=-0.414, \mathrm{P}=$ $0.0031)$ in males and positively correlated with ALB $(r=0.377, P=0.048)$, and negatively correlated with IL-6 ( $r=-0.484, P=0.012)$ in females. Multivariate analysis adjusted for these items, age, and BMI showed a significant difference in male GDF-15 ( $P=0.011$, OR: $7.86,95 \% \mathrm{Cl}: 1.09-56.58)$. Therefore, GDF-15 in male patients is considered a biomarker of low SMI.

\section{Introduction}

Sarcopenia was proposed by Rosenberg in 1989 as a term for age-related muscle loss[1]. Subsequently, in the European Working Group on Sarcopenia in Older People (EWGSOP), sarcopenia related to aging was classified as primary, and activity, disease, and nutrition-related sarcopenia were classified as secondary[2]. In many research groups, such as the Asian Working Group for Sarcopenia (AWGS), the diagnostic criteria for sarcopenia require either low muscle strength (MS) or low physical function in addition to low skeletal muscle mass index (SMI)[3].

Young people with Crohn's disease (CD) often experience abdominal pain, diarrhea, and weight loss, and $\mathrm{CD}$ is a debilitating disease of unknown cause. Curative treatment cannot be expected for CD. Medical treatment such as anti-tumor necrosis factor alpha (TNFa) antibody is the main treatment; however, surgical treatment is required for intestinal complications such as severe stenosis and fistula. In most of the previous reports on CD, sarcopenia was often defined only by SMl; a recent meta-analysis[4] reported that $52 \%$ of patients with CD were defined by SMI. Bryant et al. demonstrated that the prevalence of sarcopenia defined by SMI and grip strength was $12 \%$ in patients with $\mathrm{CD}$ [5]. However, no report to date has demonstrated the prevalence of sarcopenia diagnosed by the appropriate definition in Japanese patients with $\mathrm{CD}$.

The cumulative operation rates for CD are approximately 50\%, 10 years after onset[6], and cumulative reoperation rates after intestinal resection are $22-28 \%$ and $30-40 \% 5$ and 10 years after surgery, respectively[7]. Recurrence at the anastomotic site under endoscopic observation is even higher, reported to be $70-90 \%$ at 1 year after surgery $[8,9]$. Therefore, intestinal resection should be avoided as much as possible because $C D$ is prone to early postoperative recurrence. Bamba et al. reported that sarcopenia, defined by SMI, is a predictor of intestinal resection in $\mathrm{CD}[10]$, and there are several reports on the 
relationship between SMI and surgery, such as the association between low SMI and postoperative complications in $\mathrm{CD}[11]$.

Therefore, low SMI is an important problem in patients with CD who are considered for intestinal resection. SMI was measured using dual energy X-ray absorptiometry (DXA) or bioelectrical impedance analysis (BIA); however, both require the purchase of expensive measuring equipment. Computed tomography (CT) is also used, but it requires special software and has the disadvantage of exposing the patient to radiation, making it difficult to perform many times. For this reason, facilities that can measure SMI are limited.

Because various mechanisms contribute to sarcopenia, there are various reports on biomarkers that can help in the diagnosis of sarcopenia. Franscesco et al. summarized previous reports on biomarkers of sarcopenia by mechanism[12]. For example, albumin (ALB), a behavior-mediated pathway, is an indicator of nutritional status, and undernutrition can be an indicator of sarcopenia[13]. Growth differentiation factor-8 (GDF-8) and growth differentiation factor-15 (GDF-15) are factors that suppress muscle growth[14, 15]. Interleukin-6 (IL-6) and TNF-a are inflammation-mediated pathways that act as receptors in skeletal muscle and promote muscle atrophy[16]. If a biomarker that predicts low SMI in CD patients can be identified, it will be useful for daily medical care.

The purpose of this study is to first reveal the prevalence of "true sarcopenia" assessed as both the MS and SMI in Japanese patients with CD. The second purpose was to search for biomarkers that could be indicators of low SMI.

\section{Methods}

\section{Study design and patients}

We performed a cross-sectional study of 78 consecutive outpatients or inpatients with CD who visited the Department of Gastroenterology, Nagasaki University Hospital, between August 2018 and July 2020. Our research protocol complied with the guidelines of the Declaration of Helsinki and was approved by the Nagasaki University Ethics Committee (Approval No. 18121006). All patients provided informed consent in writing, and if the patients were minors, their parents consented. We excluded patients with severe CD who were difficult to move or measure physically. The diagnosis of CD was based on standardized criteria by clinical assessment, endoscopy, small bowel radiography, and histology.

\section{Clinical and laboratory data}

Patients were investigated for age, BMI, CDAl, presence or absence of biological therapy or surgery, and disease duration. Blood tests were performed on patients, and in addition to CRP and ALB, IL-6, TNF- $a$, GDF-8, and GDF-15 were measured as biomarkers. To measure GDF-8 and GDF-15 concentrations in patients' serum, we used the GDF/Myostatin Quantikine ELISA and Human GDF-15 Quantikine ELISA (R UD Systems, Minneapolis, MN, USA) according to the manufacturer's instructions. 


\section{Evaluation of SMI, MS, and sarcopenia}

In this study, sarcopenia was diagnosed using the assessment criteria for the 2019 Consensus Update on Sarcopenia Diagnosis and Treatment reported by the AWGS[3]. According to these criteria, sarcopenia was defined as low SMI and low handgrip strength. Handgrip strength was measured using a Smedley handgrip dynamometer (TTM, Tokyo, Japan) with the participant in a standing position. Two trials were performed for the right and left hands, and the two highest values were averaged and entered into the analysis. The cut-off values for low MS were $28 \mathrm{~kg}$ for men and $18 \mathrm{~kg}$ for women. The SMI was calculated using BIA (InBody 770, InBody Japan, Tokyo, Japan)[17] as the sum of the skeletal muscle mass of the arms and legs divided by the square of the individual's height $(\mathrm{kg} / \mathrm{m} 2)$. The cut-off values for low SMI were $7.0 \mathrm{~kg} / \mathrm{m}^{2}$ for males and $5.7 \mathrm{~kg} / \mathrm{m}^{2}$ for women.

If a CT scan was performed according to clinical needs within approximately 1 month after obtaining consent, the cross-sectional area of the skeletal muscles $(\mathrm{cm} 2)$ at the level of the third lumbar (L3) vertebra was measured using image analysis software (sliceOmatic V4.3, TomoVision, Magog, Quebec, Canada)[18]. SMI was calculated based on the sum of this area divided by the square of the height $\left(\mathrm{cm}^{2} / \mathrm{m}^{2}\right)$.

\section{Statistical analysis}

All statistical analyses were performed using JMP Pro version 15.0 software (SAS Institute Japan, Tokyo, Japan). A P value $<0.05$, was considered to indicate a statistically significant difference for all tests. Ttests were used to compare continuous variables, such as age, and the $\chi^{2}$ test was used to compare sex between the two groups. Correlation coefficients (Pearson correlation) were calculated for the correlation between the BIA and CT for measuring SMI and between SMI and each biomarker. If there were significant correlations between SMI and some biomarkers, we performed multivariate analysis of SMI and these biomarkers by logistic regression analysis adjusting for age and $\mathrm{BMI}$, and calculated the $\mathrm{P}$ value, ORs, and $95 \% \mathrm{Cls}$. We adjusted for age and $\mathrm{BMl}$; aging is one factor of sarcopenia, and $\mathrm{BMI}$ is correlated with SMI in IBD patients[10].

\section{Results}

\section{Prevalence of sarcopenia and low SMI}

Of the 79 patients who participated in this study, 78 patients were included in the analysis set, while those who denied the grip strength test and BIA were excluded. The clinical characteristics of the patients are shown in Table 1. Among these patients, $64.1 \%$ were male, the median age was $42(31.8-51)$ years, the median body mass index (BMI) was $19.9(18.6-21.7) \mathrm{kg} / \mathrm{m}^{2}$, the median disease duration was 12.29 (6.44-19) years, and Crohn's disease activity index (CDAl) was 84.5 (53.5-119). Sarcopenia, which met the criteria for low MS and low SMI, was found in six patients (8\%). Low SMI was found in 33 patients (42.3\%). 
Table 1

Patients' clinical characteristics

\begin{tabular}{|c|c|c|c|}
\hline & Male $(n=50)$ & Female $(n=28)$ & Total $(n=78)$ \\
\hline Age (years), median (IQR) & $43(33-51.3)$ & $39(29.5-48.5)$ & $42(31.8-51)$ \\
\hline $\begin{array}{l}\text { Body mass index }\left(\mathrm{kg} / \mathrm{m}^{2}\right) \text {, } \\
\text { median (IQR) }\end{array}$ & $20(18.7-21.9)$ & $19.4(18.4-21.3)$ & $19.9(18.6-21.7)$ \\
\hline Height (cm), median (IQR) & $170.2(166-174.8)$ & $\begin{array}{l}157.6(152.3- \\
162.0)\end{array}$ & $\begin{array}{l}166(159.0- \\
173.8)\end{array}$ \\
\hline Weight (kg), median (IQR) & $58.2(53.0-63.8)$ & $49.2(42.6-54.6)$ & $55.5(49.3-62.0)$ \\
\hline Grip strength (kg), median (IQR) & $37.3(32.7-42.3)$ & $23.9(19.8-26.8)$ & $32.9(25.8-40.8)$ \\
\hline $\begin{array}{l}\text { Positive for handgrip strength } \\
\text { decline (\%) }\end{array}$ & $6(12)$ & $1(4)$ & $7(9.3)$ \\
\hline $\mathrm{SMI}\left(\mathrm{kg} / \mathrm{m}^{2}\right)$, median (IQR) & $7.3(6.7-7.5)$ & $5.5(5.1-6.1)$ & $6.7(6.0-7.4)$ \\
\hline Positive for SMI decline (\%) & $17(34)$ & $16(57.1)$ & $33(42.3)$ \\
\hline With Sarcopenia (\%) & $5(10)$ & $1(4)$ & $6(8)$ \\
\hline Biological therapy (for CD) (\%) & $46(92)$ & $23(82.1)$ & $69(88.5)$ \\
\hline $\begin{array}{l}\text { Disease duration (year), median } \\
\text { (IQR) }\end{array}$ & $14(6.74-20.65)$ & $\begin{array}{l}11.25(6.15- \\
17.98)\end{array}$ & $12.29(6.44-19)$ \\
\hline CDAl, median (IQR) & $83(52-125)$ & $86(60.5-114.5)$ & $84.5(53.5-119)$ \\
\hline CRP (mg/dL), median (IQR) & $0.2(0.06-0.37)$ & $0.1(0.04-0.32)$ & $0.13(0.05-0.35)$ \\
\hline Alb (g/dL), median (IQR) & $3.8(3.5-4.3)$ & $4.1(3.9-4.4)$ & $4(3.7-4.4)$ \\
\hline IL-6 (pg/mL), median (IQR) & $1.96(1.31-3.83)$ & $1.10(0.80-2.37)$ & $1.64(0.92-3.38)$ \\
\hline TNF-a (pg/mL), median (IQR) & $9.46(4.46-39.65)$ & $8.4(5.05-50.65)$ & $8.49(4.46-47.5)$ \\
\hline GDF-8 (pg/mL), median (IQR) & $\begin{array}{l}4142.7(3310.6- \\
6019.8)\end{array}$ & $\begin{array}{l}3371.7(2820.2- \\
4061.3)\end{array}$ & $\begin{array}{l}3808(3174.8- \\
5282.6)\end{array}$ \\
\hline GDF-15 (pg/mL), median (IQR) & $\begin{array}{l}795.1(358.5- \\
1494)\end{array}$ & $\begin{array}{l}349.3(282.6- \\
831.8)\end{array}$ & $\begin{array}{l}477.1(296.3- \\
1134.22)\end{array}$ \\
\hline \multicolumn{4}{|c|}{$\begin{array}{l}\text { BMI, body mass index; SMI, skeletal muscle mass index; CDAl, Crohn's disease activity index; CRP, C- } \\
\text { reactive protein; ALB, albumin; IL-6, Interleukin-6; TNF-a, tumor necrosis factor alpha; GDF-8, growth } \\
\text { differentiation factor-8; GDF-15, growth differentiation factor-15; IQR, interquartile range }\end{array}$} \\
\hline
\end{tabular}

We also investigated the correlation of SMI between CT and BIA in 13 patients who underwent CT within a month of BIA (Fig. 1). The Pearson correlation test showed a highly significant correlation $(r=0.83, P=$ 0.0003). 


\section{Comparison of the sarcopenia group and the non- sarcopenia group}

The characteristics of the patients who met the criteria for sarcopenia are showed in Table 2. Ten percent of men $(5 / 50)$ and $3.6 \%$ of women $(1 / 28)$ were diagnosed with sarcopenia. In the sarcopenia group, there was a tendency for long-term disease duration $(20.02 \pm 12.0$ years, $P=0.14)$ and short stature $(160.5 \pm 8.3$ $\mathrm{cm}, \mathrm{P}=0.13)$, but there was no significant difference. Age, $\mathrm{BMI}$, and CDAl were also not significant indicators of sarcopenia.

Table 2

Comparison of sarcopenia group and non-sarcopenia group

\begin{tabular}{|c|c|c|c|}
\hline & Sarcopenia $(n=6)$ & Non-sarcopenia $(n=72)$ & $P$ value \\
\hline Male/Female & $5 / 1$ & $45 / 27$ & 0.31 \\
\hline Age (years), mean $\pm S D$ & $48.5 \pm 19.2$ & $42.1 \pm 12.9$ & 0.26 \\
\hline $\mathrm{BMI}\left(\mathrm{kg} / \mathrm{m}^{2}\right)$, mean $\pm \mathrm{SD}$ & $20.0 \pm 3.48$ & $20.5 \pm 3.2$ & 0.69 \\
\hline Height $(\mathrm{cm})$, mean $\pm S D$ & $160.5 \pm 8.27$ & $166.0 \pm 8.4$ & 0.13 \\
\hline Weight $(\mathrm{kg})$, mean \pm SD & $51.8 \pm 12.6$ & $56.7 \pm 10.9$ & 0.31 \\
\hline Disease duration (year), mean \pm SD & $20.02 \pm 12.00$ & $13.65 \pm 10.02$ & 0.14 \\
\hline CDAl, mean \pm SD & $89.8 \pm 39.9$ & $95.7 \pm 57.2$ & 0.81 \\
\hline
\end{tabular}

Because the number of patients with sarcopenia was too low for analysis, we divided the patients into two subgroups according to the SMI value (low SMI group and normal SMI group). The characteristics of the two groups are shown in Table 3. The number of females was significantly higher than that of males in the low SMI group (57.1\%, $\mathrm{P}=0.047)$. BMI $\left(18.8 \pm 2.3 \mathrm{~kg} / \mathrm{m}^{2}, \mathrm{P}<0.0001\right)$, height $(162.5 \pm 8.5 \mathrm{~cm}, \mathrm{P}=$ $0.006)$, and weight $(50.0 \pm 8.8 \mathrm{~kg}, \mathrm{P}<0.0001)$ were also significantly higher in the low SMI group. However, there were no significant differences in age, CDAl, or disease duration. 
Table 3

Comparisons between the low SMI group and normal group

\begin{tabular}{|c|c|c|c|}
\hline & Low SMI (n = 33) & Normal $(n=45)$ & $P$ value \\
\hline Male/Female & $17 / 16$ & $33 / 12$ & 0.047 \\
\hline Age (year), mean \pm SD & $44.0 \pm 14.6$ & $41.6 \pm 12.6$ & 0.47 \\
\hline BMI $\left(\mathrm{kg} / \mathrm{m}^{2}\right)$, mean $\pm \mathrm{SD}$ & $18.8 \pm 2.3$ & $21.6 \pm 3.2$ & $<.0001$ \\
\hline Height $(\mathrm{cm})$, mean \pm SD & $162.5 \pm 8.5$ & $167.8 \pm 7.8$ & 0.006 \\
\hline Weight $(\mathrm{kg})$, mean \pm SD & $50.0 \pm 8.8$ & $61.0 \pm 10.2$ & $<.0001$ \\
\hline CDAl, mean \pm SD & $97.6 \pm 59.5$ & $93.4 \pm 53.6$ & 0.76 \\
\hline Disease duration (year), mean $\pm S D$ & $15.9 \pm 12.06$ & $12.84 \pm 8.57$ & 0.21 \\
\hline $\mathrm{CRP}(\mathrm{mg} / \mathrm{dL})$, mean $\pm \mathrm{SD}$ & $0.21 \pm 0.31$ & $0.39 \pm 0.51$ & 0.09 \\
\hline $\operatorname{ALB}(\mathrm{g} / \mathrm{dL})$, mean $\pm \mathrm{SD}$ & $3.91 \pm 0.56$ & $3.92 \pm 0.54$ & 0.91 \\
\hline $\mathrm{IL}-6(\mathrm{pg} / \mathrm{mL})$, mean $\pm \mathrm{SD}$ & $2.69 \pm 2.61$ & $2.41 \pm 2.07$ & 0.6 \\
\hline TNF-a $(p g / m L)$, mean \pm SD & $17.69 \pm 20.45$ & $28.63 \pm 34.9$ & 0.09 \\
\hline GDF-8 (pg/mL), mean \pm SD, & $4107.1 \pm 1418.1$ & $4244.9 \pm 1558.2$ & 0.7 \\
\hline GDF-15 (pg/mL), mean \pm SD & $1511.0 \pm 1646.3$ & $688.2 \pm 575.3$ & 0.013 \\
\hline
\end{tabular}

As for biomarkers, GDF-15 (1511.0 $\pm 1646.3 \mathrm{pg} / \mathrm{mL}, \mathrm{P}=0.013)$ was significantly higher in the low SMI group. The level of C-reactive protein (CRP) $(0.21 \pm 0.31 \mathrm{mg} / \mathrm{dl}, \mathrm{P}=0.09)$ and TNF-a $(17.69 \pm 20.45 \mathrm{pg} / \mathrm{mL}$, $P=0.09)$ tended to be lower in the low SMI group, but there were no significant differences. No differences were found in other biomarkers, including ALB, IL-6, and GDF-8.

\section{Examination of biomarkers separated by sex}

Because sex affects the frequency of low SMI or the value of some biomarkers, we examined the biomarkers associated with SMI by sex. The correlations between SMI and each biomarker in men are shown in Table 4. The value of GDF-15 $(r=-0.410, P=0.0034)$ was negatively correlated with $S M I$ in men. CRP, ALB, IL-6, TNFa, and GDF-8 levels were not correlated with SMI. 
Table 4

Correlation with SMI in males

\begin{tabular}{|lll|}
\hline & $\mathbf{r}$ & P-value \\
\hline CRP & 0.0623 & 0.67 \\
\hline ALB & 0.132 & 0.37 \\
\hline IL-6 & -0.242 & 0.094 \\
\hline TNFa & 0.163 & 0.262 \\
\hline GDF-8 & 0.153 & 0.29 \\
\hline GDF-15 & -0.414 & 0.0031 \\
\hline $\begin{array}{l}\text { CRP, C-reactive protein; ALB, albumin; IL-6, Interleukin-6; TNF-a, tumor necrosis factor alpha; GDF-8, } \\
\text { growth differentiation factor-8; GDF-15, growth differentiation factor-15 }\end{array}$ \\
\hline
\end{tabular}

In females, the correlation between SMI and each biomarker is shown in Table 5. The value of ALB ( $r=$ $0.377, \mathrm{P}=0.048)$ was positively correlated while that of $\mathrm{IL}-6(\mathrm{r}=-0.484, \mathrm{P}=0.012)$ was negatively correlated with SMI in women.

Table 5

Correlation with SMI in females

\begin{tabular}{|lll|}
\hline & $\mathbf{r}$ & P-value \\
\hline CRP & 0.0637 & 0.75 \\
\hline ALB & 0.377 & 0.048 \\
\hline IL-6 & -0.484 & 0.012 \\
\hline TNFa & -0.011 & 0.96 \\
\hline GDF-8 & 0.0993 & 0.63 \\
\hline GDF-15 & -0.248 & 0.22 \\
\hline $\begin{array}{l}\text { CRP, C-reactive protein; ALB, albumin; IL-6, Interleukin-6; TNF-a, tumor necrosis factor alpha; GDF-8, } \\
\text { growth differentiation factor-8; GDF-15, growth differentiation factor-15 }\end{array}$ \\
\hline
\end{tabular}

CRP, TNFa, GDF-8, and GDF-15 levels did not correlate with SMI. Because GDF-15 in men and ALB and IL6 in women were significantly correlated with SMI, we performed multivariate analysis of these biomarkers adjusted for age and sex by logistic regression analysis. In males, shown in Table 6, the high value of GDF-15 ( $\mathrm{P}=0.017$; OR 7.86; $95 \% \mathrm{Cl} 1.09-56.58)$ was an independent factor for low SMI. The area under the receiver operating characteristic curve was 0.755 , the cutoff value was $940.11 \mathrm{pg} / \mathrm{ml}$, the sensitivity was 0.75 , and the specificity was 0.76 . In females, both the low value of ALB $(P=0.47)$ and the high value of IL-6 $(P=0.26)$ did not significantly contribute to low SMI. 
Table 6

Factors associated with low SMI among male CD patients

\begin{tabular}{|lllllll|}
\hline \multicolumn{3}{|c|}{ Univariate } & \multicolumn{5}{c|}{ Multivariate } \\
\hline Covariate & P value & OR & $95 \% \mathrm{Cl}$ & P value & OR & $95 \% \mathrm{Cl}$ \\
\hline Age & 0.02 & 1.06 & $\mathbf{1 . 0 1 - 1 . 1 2}$ & 0.26 & 1.04 & $0.97-1.11$ \\
\hline GDF-15 & 0.0006 & 1.001 & $1.0002-1.002$ & 0.011 & 1.001 & $1.00006-1.0017$ \\
\hline BMI & 0.021 & 0.76 & $0.57-0.997$ & 0.049 & 0.75 & $0.54-1.03$ \\
\hline GDF-15, growth differentiation factor-15; BMl, body mass index; OR, odds ratio; Cl, confidence interval \\
\hline
\end{tabular}

\section{Discussion}

This cross-sectional study demonstrated the frequency of sarcopenia using an appropriate definition, incorporating the assessment of both muscle mass and strength. Overall, $42.3 \%$ of Japanese patients with $\mathrm{CD}$ had a low SMI and 7.7\% were diagnosed with sarcopenia. The median age of this CD cohort was 42 years, and $89 \%$ of the patients were aged under 60 years. Although we have no data on healthy controls of the same age as this cohort, the frequency of sarcopenia in CD was about the same as that in healthy individuals over the age of 60 years, which was reported to be $8.2 \%$ by a recent survey[19].

Although CT is well recognized as the gold standard investigative tool for SMI, it involves radiation exposure and is costly for patients. Moreover, CT information can only be obtained when CT examination is necessary in clinical practice. Most previous reports regarding sarcopenia in CD used CT to estimate $\mathrm{SMI}$ and were analyzed retrospectively. The patient groups in those studies were biased towards the collection of patients who required CT examination in their practice. In this study, we showed that the BIA measures of SMI were strongly correlated with CT-based estimates. Thus, BIA is also useful for measuring muscle mass in patients with CD. BIA should be actively used in future prospective studies on sarcopenia in $\mathrm{CD}$ because it is non-invasive and convenient.

GDF-15 is a member of the transforming growth factor-beta superfamily (TGF- $\beta$ s) and is expressed at low concentrations in various organs such as the liver, kidneys, lungs, and other tissues under normal physiological conditions[20,21]. Elevated inflammatory cytokines, oxidative stress, and hypoxia are known to elevate GDF-15 expression. We demonstrated that GDF-15 negatively correlates with SMI in male patients with CD and can be an indicator of low SMI in multivariate analysis adjusted for age and BMI. The inverse correlation between muscle mass and circulating GDF-15 levels is not specific to CD and has been reported in patients with chronic obstructive pulmonary disease[22], pulmonary arterial hypertension[23], and preoperative cardiovascular disease[24]. Moreover, an inverse association between GDF-15 and MS or function has also been reported in patients with cardiometabolic disease[25] and cancer[26], and in healthy community-dwelling adults[27]. Although the exact mechanisms of serum GDF15 elevation in CD patients with low SMI remain unknown, some evidence suggests that GDF-15 may 
promote muscle wasting. It has been reported that glial cell-derived neurotrophic factor receptor alpha-like (GFRAL), the receptor for GDF-15, is present in the brainstem, and that binding of GDF-15 leads to loss of appetite and weight[28-31]. In addition, several reports have suggested a direct catabolic effect of GDF15 on muscle mass. Patel et al.[22] demonstrated that local overexpression of GDF-15 leads to a reduction in the fiber size of the tibialis anterior muscle in mice. Furthermore, genetic loss of GDF-15 did not affect muscle wasting in transgenic mice characterized by mitochondrial stress-driven skeletal muscle atrophy[32]. In vitro, GDF-15 treatment of C2C12 myotubes elevated the mRNA expression of muscle atrophy-related genes, such as MuRF-1 and Atrogin, and downregulated the expression of muscle microRNAs, such as miR-1, miR-133a, and miR-181a[33]. Further studies are needed to clarify the relationship between circulating GDF-15 levels and muscle wasting in patients with CD and sarcopeniarelated outcomes.

Our study has several limitations. First, in our study, sarcopenia was evaluated by MS and SMI. It did not include physical function such as the $6 \mathrm{~m}$ walking speed or sit-to-stand test. Therefore, if we evaluated physical function as well as MS, we might have been able to indicate the rate of sarcopenia more accurately in CD patients and analyze between the sarcopenia and non-sarcopenia groups. Second, because our study was a single-center, cross-center study, the causal relationship between GDF-15 and SMI in male patients with CD was unclear. In addition, because there were relatively more males in our study population, it was possible that there was a sex difference in GDF-15 due to bias of sex-specific effects. Therefore, further investigation is needed to clarify the relationship between GDF-15 and SMI in patients with CD. Despite these limitations, to our knowledge, this study is the first to evaluate the association between SMI in CD and GDF-15. We hope that GDF-15 will be a target for indicators of low $\mathrm{SMI}$ and therapeutic effects in patients with $\mathrm{CD}$ through the accumulation of case data in the future.

In conclusion, $42.3 \%$ of Japanese patients with CD had a low SMI and 7.7\% were diagnosed with sarcopenia. A low SMI was associated with GDF-15 in male patients with CD. Measurement of GDF-15 may be a predictive biomarker for low SMI, leading to poor prognosis in patients with CD.

\section{Declarations}

\section{Acknowledgements}

We are thankful for the cooperation of Eriko Nakao with measurements of SMI using Inbody and handgrip strength, and Yasuko Kanda and Mami Morishita with measurements of GDF-8 and GDF-15.

\section{Authors' contributions}

$\mathrm{HY}, \mathrm{FT}$, and $\mathrm{MH}$ wrote the manuscript and designed the protocol. $\mathrm{HY}$ and $\mathrm{MH}$ performed the statistical analyses. $\mathrm{HY}, \mathrm{FT}, \mathrm{KM}, \mathrm{MK}, \mathrm{YA}, \mathrm{KO}, \mathrm{MT}, \mathrm{KH}$, and NY collected the patient data. KN finalized the manuscript. All the authors contributed to the study concept and design.

\section{Competing interests}

Page $11 / 15$ 
The authors declare no competing interests.

\section{Data availability}

All data generated or analyzed in this study are included in this published article.

\section{References}

1. Rosenberg, I. H. Sarcopenia: origins and clinical relevance. J. Nutr. 127(5) Suppl, 990S-991S(1997). 10.1093/jn/127.5.990S, Pubmed:9164280.

2. Cruz-Jentoft, A. J. et al. Sarcopenia: European consensus on definition and diagnosis: report of the European Working Group on Sarcopenia in Older People. Age Ageing 39, 412-423(2010). 10.1093/ageing/afq034, Pubmed:20392703.

3. Chen, L. K. et al. Asian Working Group for Sarcopenia. Asian Working Group for Sarcopenia: 2019 Consensus update on sarcopenia diagnosis and treatment. J. Am. Med. Dir. Assoc, 21, 300-3072 https://doi.org/10.1016/j.jamda.2019.12.012 (2020).

4. Ryan, E. et al. Sarcopenia and inflammatory bowel disease: A systematic review. Inflamm. Bowel Dis. 25, 67-73(2019). 10.1093/ibd/izy212, Pubmed:29889230.

5. Bryant, R. V. et al. Low muscle mass and sarcopenia: common and predictive of osteopenia in inflammatory bowel disease. Aliment. Pharmacol. Ther, 41, 895-906 https://doi.org/10.1111/apt.13156 (2015).

6. Peyrin-Biroulet, L., Loftus, E. V. Jr., Colombel, J. F. \& Sandborn, W. J. The natural history of adult Crohn's disease in population-based cohorts. Am. J. Gastroenterol, 105, 289-297 https://doi.org/10.1038/ajg.2009.579 (2010).

7. Ozuner, G., Fazio, V. W., Lavery, I. C., Milsom, J. W. \& Strong, S. A. Reoperative rates for Crohn's disease following strictureplasty. Long-term analysis. Dis. Colon Rectum 39, 1199-1203(1996). 10.1007/BF02055108, Pubmed:8918424.

8. Rutgeerts, P. et al. Predictability of the postoperative course of Crohn's disease., 99, 956-963 (1990). 10.1016/0016-5085(90)90613-6, Pubmed:2394349.

9. Olaison, G., Smedh, K. \& Sjödahl, R. Natural course of Crohn's disease after ileocolic resection: endoscopically visualised ileal ulcers preceding symptoms., 33, 331-335 https://doi.org/10.1136/gut.33.3.331 (1992).

10. Bamba, S. et al. Sarcopenia is a predictive factor for intestinal resection in admitted patients with Crohn's disease. PLOS ONE 12, e0180036(2017). 10.1371/journal.pone.0180036, Pubmed:28644887.

11. Zager, Y. et al. Low psoas muscle area is associated with postoperative complications in Crohn's disease. Int. J. Colorectal Dis. 36, 543-550(2021). 10.1007/s00384-020-03799-1, Pubmed:33236229. 
12. Curcio, F. et al. Biomarkers in sarcopenia: A multifactorial approach. Exp. Gerontol. 85, 1-8(2016). 10.1016/j.exger.2016.09.007, Pubmed:27633530.

13. Schalk, B. W., Deeg, D. J., Penninx, B. W., Bouter, L. M. \& Visser, M. Serum albumin and muscle strength: a longitudinal study in older men and women. J. Am. Geriatr. Soc. 53, 1331-1338(2005). 10.1111/j.1532-5415.2005.53417.x, Pubmed:16078958.

14. Léger, B., Derave, W., De Bock, K., Hespel, P. \& Russell, A. P. Human sarcopenia reveals an increase in SOCS-3 and myostatin and a reduced efficiency of Akt phosphorylation. Rejuvenation Res. 11, 163175B(2008). 10.1089/rej.2007.0588, Pubmed:18240972.

15. Bloch, S. A. et al. Sustained elevation of circulating growth and differentiation factor-15 and a dynamic imbalance in mediators of muscle homeostasis are associated with the development of acute muscle wasting following cardiac surgery. Crit. Care Med, 41, 982-989 https://doi.org/10.1097/CCM.0b013e318274671b (2013).

16. Petersen, A. M. \& Pedersen, B. K. The anti-inflammatory effect of exercise. J. Appl. Physiol. (1985) 98, 1154-1162(2005). 10.1152/japplphysiol.00164.2004, Pubmed:15772055.

17. Lee, S. Y. et al. Comparison between Dual-Energy X-ray absorptiometry and bioelectrical impedance analyses for accuracy in measuring whole body muscle mass and appendicular skeletal muscle mass. Nutrients 10(2018). 10.3390/nu10060738, Pubmed:29880741.

18. Mitsiopoulos, N. et al. Cadaver validation of skeletal muscle measurement by magnetic resonance imaging and computerized tomography. J. Appl. Physiol. (1985), 85, 115-122 https://doi.org/10.1152/jappl.1998.85.1.115 (1998).

19. Yoshimura, N. et al. Is osteoporosis a predictor for future sarcopenia or vice versa? Four-year observations between the second and third ROAD study surveys. Osteoporos Int 28, 189-199(2017). 10.1007/s00198-016-3823-0, Pubmed:27885410.

20. Tsai, V. W., Lin, S., Brown, D. A., Salis, A. \& Breit, S. N. Anorexia-cachexia and obesity treatment may be two sides of the same coin: role of the TGF-b superfamily cytokine MIC-1/GDF15. Int. J. Obes. (Lond), 40, 193-197 https://doi.org/10.1038/ijo.2015.242 (2016).

21. Breit, S. N. et al. The TGF- $\beta$ superfamily cytokine, MIC-1/GDF15: a pleotrophic cytokine with roles in inflammation, cancer and metabolism., 29, 187-195 https://doi.org/10.3109/08977194.2011.607137 (2011).

22. Patel, M. S. et al. Growth differentiation factor- 15 is associated with muscle mass in chronic obstructive pulmonary disease and promotes muscle wasting in vivo. J. Cachexia Sarcopenia Muscle, 7, 436-448 https://doi.org/10.1002/jcsm.12096 (2016).

23. Garfield, B. E. et al. Growth/differentiation factor 15 causes TGF $\beta$-activated kinase 1-dependent muscle atrophy in pulmonary arterial hypertension. Thorax 74, 164-176(2019). 10.1136/thoraxjnl2017-211440, Pubmed:30554141.

24. Nakajima, T. et al. Growth differentiation Factor-15 (GDF-15) is a biomarker of muscle wasting and renal dysfunction in preoperative cardiovascular surgery patients. J. Clin. Med. 8(2019). 10.3390/jcm8101576, Pubmed:31581569. 
25. Oba, K. et al. Serum growth differentiation factor 15 level is associated with muscle strength and lower extremity function in older patients with cardiometabolic disease. Geriatr. Gerontol. Int, 20, 980-987 https://doi.org/10.1111/ggi.14021 (2020).

26. Lerner, L. et al. Plasma growth differentiation factor 15 is associated with weight loss and mortality in cancer patients. J. Cachexia Sarcopenia Muscle, 6, 317-324 https://doi.org/10.1002/jcsm.12033 (2015).

27. Semba, R. D. et al. Elevated plasma growth and differentiation Factor 15 is associated With slower gait speed and lower physical performance in healthy community-dwelling adults. J. Gerontol. A Biol. Sci. Med. Sci. 75, 175-180(2020). 10.1093/gerona/glz071, Pubmed:30874790.

28. Yang, L. et al. GFRAL is the receptor for GDF15 and is required for the anti-obesity effects of the ligand. Nat. Med, 23, 1158-1166 https://doi.org/10.1038/nm.4394 (2017).

29. Mullican, S. E. et al. GFRAL is the receptor for GDF15 and the ligand promotes weight loss in mice and nonhuman primates. Nat. Med, 23, 1150-1157 https://doi.org/10.1038/nm.4392 (2017).

30. Emmerson, P. J. et al. The metabolic effects of GDF15 are mediated by the orphan receptor GFRAL. Nat. Med, 23, 1215-1219 https://doi.org/10.1038/nm.4393 (2017).

31. Hsu, J. Y. et al. Non-homeostatic body weight regulation through a brainstem-restricted receptor for GDF15. Nature 550, 255-259(2017). 10.1038/nature24042, Pubmed:28953886.

32. Ost, M. et al. Muscle-derived GDF15 drives diurnal anorexia and systemic metabolic remodeling during mitochondrial stress. EMBO Rep, 21, e48804 (2020).

33. Bloch, S. A. et al. Increased expression of GDF-15 may mediate ICU-acquired weakness by downregulating muscle microRNAs. Thorax 70, 219-228(2015). 10.1136/thoraxjnl-2014-206225, Pubmed:25516419.

\section{Figures}




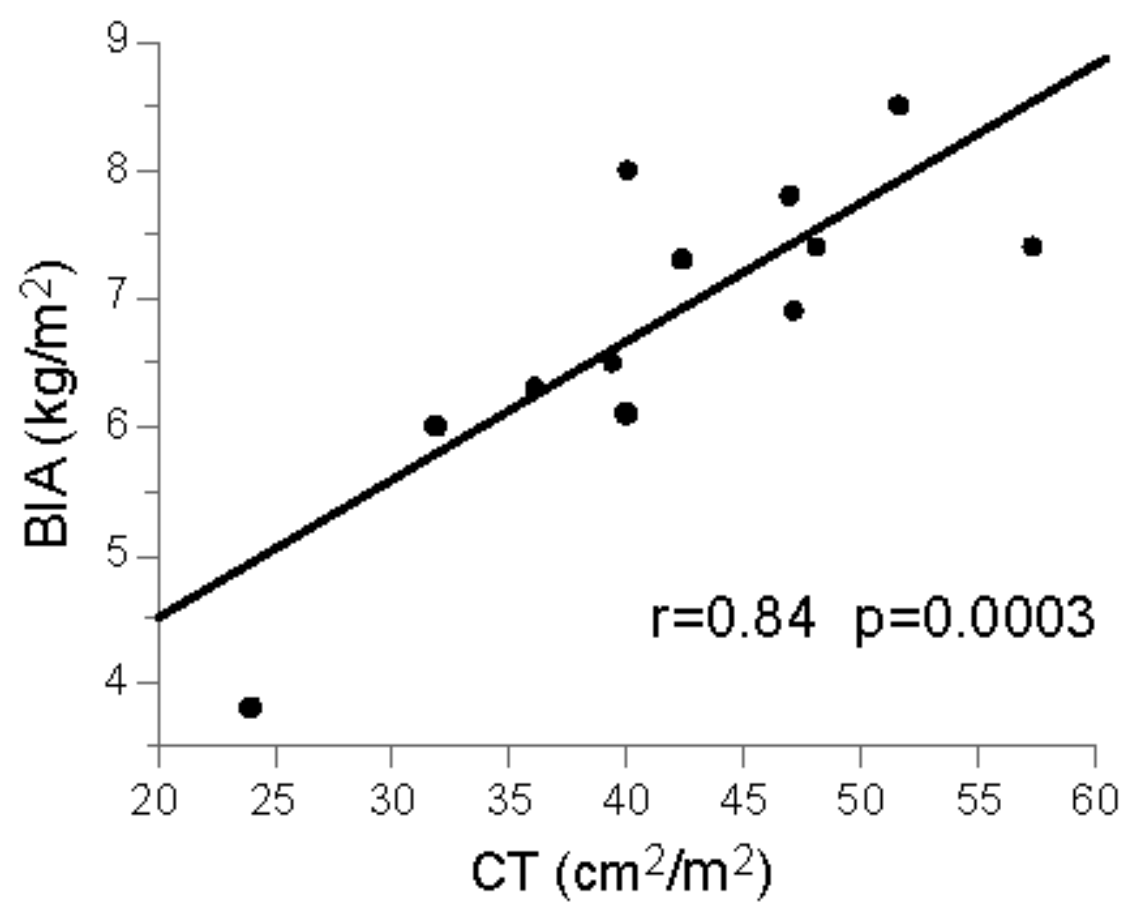

Figure 1

Correlation with SMI between the CT and BIA method. SMI, skeletal muscle mass index; BIA, bioelectrical impedance analysis; $\mathrm{CT}$, computed tomography. 